\title{
Anatomical variations of the sciatic nerve: A descriptive study
}

\author{
Chandrasiri DACT ${ }^{1}$, Patabandige $\mathrm{DJ}^{1}$, Ekanayaka EADA ${ }^{1}$, Dissanayaka $\mathrm{JK}^{1}$ \\ ${ }^{I}$ Department of Anatomy, Faculty of Medicine, University of Peradeniya. \\ *dactchandrasisi@gmail.com
}

The sciatic nerve (SN) (L4-S3) usually leaves the pelvis below piriformis and descends, dividing into the tibial nerve (TN) and common peroneal nerve $(\mathrm{CPN})$ at varying levels proximal to the knee. Understanding about these variations is important in clinical medicine and for the knowledge in anatomy. Here we present the variations of high and low divisions of the SN. Twenty seven cadavers (53 limbs) at the Department of Anatomy, Faculty of Medicine University of Peradeniya, Sri Lanka, which were used for routine undergraduate dissections were studied, and the course of the SN with the levels of bifurcation were quantitatively documented from the midpoint of the two femoral condyles to the point of division of the nerve. Of the 53 limb specimens studied, the division was in the normal confinements in 24 (45.28\%) limbs. In $14(26.42 \%)$, there was high division of $\mathrm{SN}$, and in another $14(26.42 \%)$, there was low division. In 01 (1.88\%) case, the CPN was emerging through the piriformis muscle to begin with, while the TN was emerging below PM. The knowledge on abnormal variations of the $\mathrm{SN}$ is known for causing nondiscogenic sciatica with sciatic nerve compression, especially piriformis syndrome when it traverses through the piriformis muscle. Even though the lower division of the $\mathrm{SN}$ is relatively uncommon, we found it rather in higher percentages, as well as its high division of SN. This knowledge is useful in pure anatomical point of view as well as in clinical medicine. 